\title{
A TOPOLOGICAL INTERPRETATION FOR THE BIAS INVARIANT
}

\author{
MICHEAL DYER
}

\begin{abstract}
The bias invariant has been used to distinguish between the homotopy types of 2-complexes. In this note we show that two finite, connected 2-complexes $X$ and $Y$ with isomorphic fundamental groups and the same Euler characteristic have the same bias invariant if and only if there is a map $f: X \rightarrow Y$ which is a homology equivalence ( $\pi_{1} f$ and $H_{2} f$ are isomorphisms).
\end{abstract}

Let $G$ be a group and let $d$ be an integer $\geqslant 2$. A $[G, d]$-complex $X$ is a connected $\mathrm{CW}$-complex of dimension $\leqslant d$ whose fundamental group is isomorphic to $G$ and whose universal covering $\tilde{X}$ is $(d-1)$-connected. For any two $[G, d]$-complexes $X, X^{\prime}$ there is a comparison invariant called bias. This invariant has been used in [DS, SD, D, WB, $\mathbf{D}_{\mathbf{2}}, \mathbf{S c}, \mathbf{M}$, and $\mathbf{S}$ ] to detect when two such complexes are not homotopy equivalent.

Given any integer $d \geqslant 2$, any two $[G, d]$-complexes $X, X^{\prime}$, and any $\alpha \in$ $\operatorname{Hom}\left(\pi_{1} X, \pi_{2} X^{\prime}\right)$, there exists a continuous function $f: X \rightarrow X^{\prime}$ such that $f_{1 \#}=\alpha$. If $X, X^{\prime}$ are finite [ $\left.G, d\right]$-complexes having the same Euler characteristic, does there always exist a map $f: X \rightarrow X^{\prime}$ such that $\pi_{1} f$ and $H_{*} f$ are isomorphisms? We call such a map a homology equivalence and such spaces homology equivalent. The purpose of this note is to show that the bias detects exactly whether or not two $[G, d]$-complexes are homology equivalent. This has also been independently observed by Wolfgang Metzler.

To fix notation we observe that, if $X$ is a finite $[G, d]$-complex, then the augmented cellular chain complex $C_{*} \tilde{X} \rightarrow \mathbf{Z}$ forms a partial, finitely generated, free resolution of length $d$, called a $\left[\pi_{1} X, d\right]$-resolution. For two such complexes $X, X^{\prime}$ a comparison chain map is any chain map $k: C_{*} X \rightarrow C_{*} X^{\prime}$ which is the identity on $H_{0} C_{*} \approx \mathrm{Z}$. Any two such chain maps are chain homotopic.

If $M, F$ are free abelian groups and $i: M \neg F$ is the inclusion, then the Fox ideal $\mathscr{F}(i)$ is ideal in $\mathbf{Z}$ generated by all coordinates of the elements $i(x)$ for all $x \in M$. If one diagonalizes the matrix for $i$, call it $\operatorname{diag}(i)$; then it is easy to see that $[\mathbf{D}, 3.8]$

$$
\mathscr{F}(i)=\left\{\begin{array}{c}
\mathbf{Z} \text { if } \operatorname{diag}(i) \text { has at least one } 1 \text { or } F / M \text { has no torsion, } \\
\text { (g.c.d. }\{\text { torsion coefficients of } F / M\}) \\
\text { if } F / M \text { has torsion and } \operatorname{diag}(i) \text { has no 1's. }
\end{array}\right.
$$

We write $\mathscr{F}(i)=(m)$, and call $m$ the modulus of $i: M \rightarrow F$.

Received by the editors June 6, 1985 .

1980 Mathematics Subject Classification. Primary 57M20; Secondary 55P15.

Key words and phrases. Homotopy type of 2-complexes, homotopy type of $(\pi, m)$-complexes, bias invariant. 
For each $[G, d]$-complex $X$, we define a free abelian group $\beta \pi_{d} X \subseteq \mathbf{Z} \otimes_{G} C_{d} \tilde{X}$ to be the image of $1 \otimes j: \mathbf{Z} \otimes_{G} \pi_{d} X \rightarrow \mathbf{Z} \otimes_{G} C_{d} X$ induced by the natural inclusion $j$ : $\pi_{d} X \rightarrow C_{d} \tilde{X}$.

Fix a $[G, d]$-complex $X$ and let $(m)=\mathscr{F}(i)$ be the Fox ideal of the inclusion $i$ : $\beta \pi_{d} X \subseteq \mathbf{Z} \otimes_{G} C_{d} \tilde{X}$. Notice that the functor $\rho_{m}=\mathbf{Z}_{m} \otimes-$ has $\rho_{m}(i)=0$. Such a functor is called a "killing functor" in [D, §7].

For each $\alpha \in \operatorname{Aut}\left(\pi_{1} X\right)$, define $b(\alpha) \in \mathbf{Z}_{m}^{*} / \pm 1$ as follows. Consider the cellular chain complex $C_{*} \tilde{X} \rightarrow \mathbf{Z}$ of the universal cover $\tilde{X}$ of $X$ as a $[G, d]$-resolution by identifying $\pi_{1} X=G$. For each $\alpha$, and each $G$-module $M$, let ${ }_{\alpha} M$ denote the $G$-module with action $g * x=\alpha(g) \cdot x$ for $g \in G$ and $x \in M$. Let $i^{\prime}: \pi_{d} X \rightarrow C_{d} \tilde{X}$ be the inclusion. Let $k(X, 1 ; X, \alpha)$ be any map $k(X, 1 ; X, \alpha): \pi_{d} X \rightarrow_{\alpha} \pi_{d} X$ arising from the comparison of $C_{*} \tilde{X} \rightarrow \mathbf{Z}$ to ${ }_{\alpha} C_{*} \tilde{X} \rightarrow \mathbf{Z}$. Because the diagram

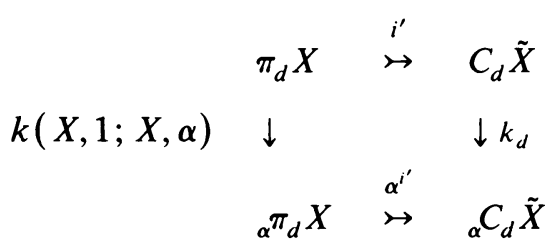

commutes, we may define $\beta(k(X, 1 ; X, \alpha)): \beta \pi_{d} X \rightarrow \beta_{\alpha} \pi_{d} X$ (functorially). Using the right exact functor $\mathbf{Z} \otimes_{G}-$, then the bias $b(X, 1 ; X, \alpha)$ is the class of $\operatorname{det}\left[\beta k(X, 1 ; X, \alpha): \beta \pi_{d} X \rightarrow \beta_{\alpha} \pi_{d} X\right]$ modulo $m$.

Similarly, if we consider ${ }_{\beta} C_{*} X^{\prime} \rightarrow \mathbf{Z}, \beta \in \operatorname{Iso}\left(G, \pi_{1} X^{\prime}\right)$, then we may define

$$
k\left(X, \alpha ; X^{\prime}, \beta\right):{ }_{\alpha} \pi_{d} X \rightarrow{ }_{\beta} \pi_{d} X^{\prime} \quad(\alpha \in \text { Aut } G)
$$

as the comparison $k$-invariant between ${ }_{\alpha} C_{*} X \rightarrow \mathbf{Z}$ and ${ }_{\beta} C_{*} X^{\prime} \rightarrow \mathbf{Z}$. Then $b\left(X, \alpha ; X^{\prime}, \beta\right) \in \mathbf{Z}_{m}^{*} / \pm 1$ is given by the class of $\operatorname{det}\left[\beta k\left(X, \alpha ; X^{\prime}, \beta\right)\right]$ (modulo $m$ ).

The definitions above are well defined because $\mathscr{F}_{m}=\mathbf{Z}_{m} \otimes\left(\mathbf{Z} \otimes_{G} \cdot\right)$ is a killing functor for $i^{\prime}: \pi_{d} X \nrightarrow C_{d} \tilde{X}[\mathbf{D}, \S 7]$. Clearly, also the Fox ideal of $\beta \pi_{d} X \nrightarrow \mathscr{F} C_{d} \tilde{X}$ is the same as $\beta_{\alpha} \pi_{d} X \nrightarrow \mathscr{F}_{\alpha} C_{d} \tilde{X}(\alpha \in$ Aut $G$ ). Arguments from $\S 7$ (or $\S 4$ ) of [D] show that $b\left(X, \alpha ; X^{\prime}, \beta\right)$ is independent of the choice of $k\left(X, \alpha ; X^{\prime}, \beta\right)$. Furthermore, it follows from 3.8 of [D] that for any $[G, d]$-complex $X^{\prime}$, the Fox ideal of $i$ : $\beta \pi_{d} X^{\prime} \nrightarrow \mathscr{F} C_{d} \tilde{X}^{\prime}$ is $(m)$, where $m$ is either 1 or the g.c.d. of the torsion coefficients of $H_{d}(G)$.

Lemma 1. Let $\alpha \in$ Aut $G$ and $\gamma \in \operatorname{Iso}\left(G, \pi_{1} X^{\prime}\right)$. Then

(a) $b\left(X, \alpha ; X^{\prime}, \gamma\right)=b\left(X, 1 ; X^{\prime}, \gamma \alpha^{-1}\right)$ and

(b) $b\left(X, 1 ; X^{\prime}, \gamma \alpha\right)=b\left(X, 1 ; X^{\prime}, \gamma\right) \cdot b(X, 1 ; X, \alpha)$.

Proof. (a) If we compare $C_{*} \tilde{X} \rightarrow \mathbf{Z}$ and ${ }_{\gamma \alpha^{-1}} C_{*} \tilde{X}^{\prime} \rightarrow \mathbf{Z}$, we obtain $k\left(X, 1 ; X^{\prime}, \gamma \alpha^{-1}\right)$. Then, applying $\alpha$ to both sequences yields ${ }_{\alpha} k\left(X, 1 ; X^{\prime}, \gamma \alpha^{-1}\right)=$ $k\left(X, \alpha ; X^{\prime}, \gamma\right)$. Hence

$$
\beta k\left(X, \alpha ; X^{\prime}, \gamma\right)=\left(\beta_{\alpha} k\left(X, 1 ; X^{\prime}, \gamma \alpha^{-1}\right)\right)=\beta k\left(X, 1 ; X^{\prime}, \gamma \alpha^{-1}\right),
$$

because $\beta_{\alpha} \pi_{d} X=\beta \pi_{d} X$, as trivial $G$-modules. Hence, $b\left(X, \alpha ; X^{\prime}, \gamma\right)=$ $b\left(X, 1 ; X^{\prime}, \gamma \alpha^{-1}\right)$. 
(b) By comparing $C_{*} \tilde{X} \rightarrow \mathbf{Z}$ to ${ }_{\alpha} C_{*} \tilde{X} \rightarrow \mathbf{Z}$ and then ${ }_{\alpha} C_{*} \tilde{X} \rightarrow \mathbf{Z}$ to ${ }_{\gamma \alpha} C_{*} \tilde{X}^{\prime} \rightarrow \mathbf{Z}$ we obtain

$$
k\left(X, 1 ; X^{\prime}, \gamma \alpha\right)=k\left(X, \alpha ; X^{\prime}, \gamma \alpha\right) k(X, 1 ; X, \alpha)
$$

and hence

$$
b\left(X, 1 ; X^{\prime}, \gamma \alpha\right)=b\left(X, 1 ; X^{\prime}, \gamma\right) b(X, 1 ; X, \alpha)
$$

by (a).

If we let $D(G, d)$ denote the image of $b$ : Aut $G \rightarrow \mathbf{Z}_{m}^{*} / \pm 1$ given by the homomorphism $\alpha \rightarrow b(X, 1 ; X, \alpha)$, we see that the comparison bias

$$
b\left(X, X^{\prime}\right)=b\left(X, 1 ; X^{\prime}, \gamma\right) \cdot D(G, d) \in \mathbf{Z}_{m}^{*} / \pm D(G, d)
$$

is independent of the choice of the "polarization" $\gamma \in \operatorname{Iso}\left(G, \pi_{1} X^{\prime}\right)$. This follows from Lemma 1(b).

THEOREM 2. For each pair of finite $[G, d]$-complexes $X, X^{\prime}$ with the same Euler characteristic, $X$ is homology equivalent to $X^{\prime}$ iff $b\left(X, X^{\prime}\right)=1$ in $\mathbf{Z}_{m}^{*} / \pm D(G, d)$.

Proof. First, we identify $\beta \pi_{d} X$ as the group $\Sigma_{d} X$ of spherical elements in $H_{d} X$; i.e. $\beta \pi_{d} X$ is the image of the Hurewicz map $h_{d}: \pi_{d} X \rightarrow H_{d} X$. Notice that any $f$ : $X \rightarrow X^{\prime}$ which induces an isomorphism $\alpha$ on $\pi_{1}$ automatically induces an isomorphism $H_{s} X \rightarrow H_{s} X^{\prime}$ for $s<d$. This follows because in these dimensions $H_{s} X \cong$ $H_{s} G$. Further, $f$ induces a commutative diagram

$$
\begin{array}{lllll}
\beta \pi_{d} X & \stackrel{i}{\hookrightarrow} & H_{d} X & \rightarrow & H_{d} G \\
\downarrow i & \downarrow \jmath & \downarrow f_{d^{*}} & & \cong \downarrow H_{d}\left(\pi_{1} f\right) \\
& \stackrel{C_{2} X}{i} & & & \\
\beta \pi_{d} X^{\prime} & \stackrel{i^{\prime}}{\rightarrow} & H_{d} X^{\prime} & \rightarrow & H_{d} G .
\end{array}
$$

Note that $H_{d} X \cong H_{d} X^{\prime}$ as finitely generated free abelian groups, because $X, X^{\prime}$ have the same Euler characteristic. The only question is, can we adjust $f$ so that $\pi_{1} f$ is still an isomorphism and $f_{d^{*}}$ is an isomorphism.

If $\Sigma_{d} X=0$, then $\beta \pi_{d} X^{\prime}=0$ also, and $f_{d^{*}}$ is an isomorphism for any $f$ with $\pi_{1} f$ an isomorphism, by the above reasoning.

Now, let us suppose that $\Sigma_{d} X \neq 0$ and that $b\left(X, X^{\prime}\right)=1$ inside $\mathbf{Z}_{m}^{*} / \pm D(G, d)$. This means there are homomorphisms $\alpha \in \operatorname{Aut} G, \beta \in \operatorname{Iso}\left(G, \pi_{1} X^{\prime}\right)$ so that

$$
b\left(X, 1 ; X^{\prime}, \beta\right) \cdot b(X, 1 ; X, \alpha)=b\left(X, 1 ; X^{\prime}, \beta \alpha\right) \equiv \pm 1 \bmod m .
$$

Let ${ }_{\beta \alpha} \pi_{d} X^{\prime}$ be denoted by $N$. We claim that there exists a map $\bar{\gamma}: C_{d} X \rightarrow \beta N$ so that $\beta k\left(X, 1 ; X^{\prime}, \beta \alpha\right)+\bar{i} \bar{\gamma}$ is an isomorphism from $\beta \pi_{d} X \rightarrow \beta N$ :

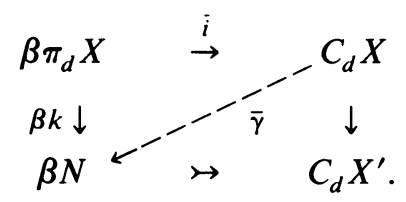


To see this, we choose bases for the free abelian groups in (4) so that $i, i^{\prime}$ are diagonal:

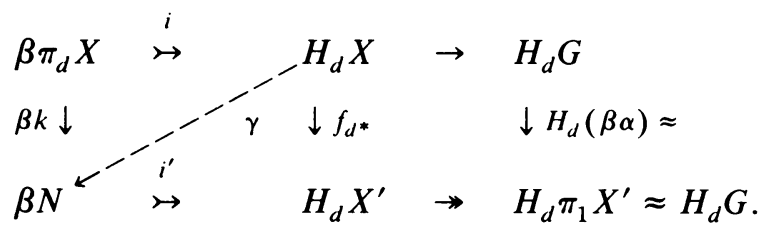

We may then canonically identify $H_{d} G=H_{d} \pi_{1} X^{\prime}$ and view $H_{d}(\beta \alpha)$ as a member of Aut $H_{d} G$, a finitely generated abelian group. By Lemma 5.1 of [D], $\operatorname{det} \beta k=$ $\pm \operatorname{det} f_{d^{*}} \equiv \pm 1 \bmod (m)$. Hence, $\operatorname{det} H_{d}(\beta \alpha) \equiv \pm 1 \bmod (m)$. Now, if $m=$ g.c.d. torsion coefficients of $H_{d} G$, we may use the argument of Theorem 6.9 of [D] (using the full strength of Appendix B) to find $\gamma: H_{d} X \rightarrow \beta N$ so that $\operatorname{det}\left(f_{d^{*}}+i^{\prime} \gamma\right)= \pm 1$. Because $H_{d} X$ is a direct summand of $C_{d} X$, let a projection $\rho$ : $C_{d} X \rightarrow H_{d} X$ be given. Then define $\bar{\gamma}=\gamma \rho$.

If $m=1$ it is because either rank $H_{d} X>$ the minimal number of generators of $H_{d} G$ or $H_{d}(G)$ is free abelian. In either case, one may choose $\gamma$ so that $\operatorname{det}\left(f_{d^{*}}+i^{\prime} \gamma\right)$ is any preassigned number; so choose $\gamma$ so that the determinant is one. Then choose $\bar{\gamma}$ as above. This finishes the proof of the claim.

Let $\mathscr{F}$ denote the functor $\mathbf{Z} \otimes_{G}-$. We observe that $q \mathscr{F}: \operatorname{Hom}_{\mathbf{Z} G}\left(C_{d} \tilde{X}, N\right) \rightarrow$ $\operatorname{Hom}_{\mathbf{z}}\left(C_{d} X, \beta N\right)$ is surjective, where $q: \mathscr{F} N \rightarrow \beta N$ is essentially $\mathscr{F}\left\{i^{\prime}: N \rightarrow C_{d} X^{\prime}\right\}$. So choose $\gamma: C_{d} \tilde{X} \rightarrow N$ so that $q \mathscr{F} \gamma=\bar{\gamma}$. Now let $k^{\prime}\left(X, 1 ; X^{\prime}, \beta \boldsymbol{\alpha}\right)=$ $k\left(X, 1 ; X^{\prime}, \beta \alpha\right)+\gamma j$, where $j: \pi_{2} X \rightarrow C_{d} \tilde{X}$ is the inclusion. But this $k^{\prime}: \pi_{d} X \rightarrow$ $\beta \alpha_{d} X^{\prime}$ is realizable as a $\pi_{d} f^{\prime}, f^{\prime}: X \rightarrow X^{\prime}$, with $\pi_{1} f^{\prime}=\beta \alpha$. (See [DS, p. 41], for a proof of the realizability of any chain map $C_{*} X \rightarrow C_{*} X^{\prime}$.) Thus $b\left(X, X^{\prime}\right)=1$ in $\mathbf{Z}_{m}^{*} / \pm D(G, d)$ implies that $X$ and $X^{\prime}$ are homology equivalent.

The converse is easy. If $f: X \rightarrow X^{\prime}$ is a map which is a homology equivalence, then $f$ induces

$$
\begin{array}{lllll}
\beta \pi_{d} X & \rightarrow & H_{d} X & \rightarrow & H_{d} G \\
\downarrow \cong \beta k\left(X, 1 ; X^{\prime}, \alpha\right) & \downarrow \cong & & \downarrow \cong \\
\beta_{\alpha} \pi_{d} X^{\prime} & \rightarrow & H_{d} X^{\prime} & \rightarrow & H_{d} G
\end{array}
$$

where $\alpha=\pi_{1} f$. Hence $\operatorname{det} \beta k\left(X, 1 ; X^{\prime}, \alpha\right)= \pm 1 ;$ so $b\left(X, 1 ; X^{\prime} ; \alpha\right)=1$ inside $\mathbf{Z}_{m}^{*} / \pm D(G, d)[\mathbf{D}, 5.1]$.

COROllaRY 5. If $X, X^{\prime}$ are finite [G,2]-complexes with the same Euler characteristic and $m=1$, then $X$ is homology equivalent to $X^{\prime}$.

This always is satisfied if $\chi(X)>\chi_{\min }(G, d)$ (= the minimal Euler characteristic for all finite $[G, d]$-complexes).

The amazing fact is that, in many cases, homology equivalence implies homotopy equivalence [ $\mathbf{D}_{\mathbf{2}}, \mathbf{D S}, \mathbf{W B}$. However, this is not the case with Dunwoody's example [Du] where $G$ is the trefoil group and $\chi(X)=\chi_{\min }(G, 2)+1$, with $X$ a [G,2]-complex. In this case $m=1$ and $H_{2} G=0$. If $Y$ is the realization of the presentation $\left\{a, b: a^{3}=b^{2}\right\}$ of $G$, then $X$ and $X^{\prime}=Y \vee S^{2}$ are homology equivalent, but not homotopy equivalent. 


\section{REFERENCES}

[SD] A. Sieradski and M. Dyer, Distinguishing arithmetic for certain stably isomorphic modules, J. Pure Appl. Algebra 15 (1979), 199-217.

[D] M. Dyer, Invariants for distinguishing between stably isomorphic modules, J. Pure Appl. Algebra 37 (1985), 117-153.

[WB] W. Browning, Finite $C W$ complexes of cohomological dimension 2 with finite abelian $\pi_{1}$, preprint, ETH, Zürich, 1979.

[Du] M. Dunwoody, The homotopy type of a two-dimensional complex, Bull. London Math. Soc. 8 (1976), 282-285.

[ $\mathbf{D}_{2}$ ] M. Dyer, Homotopy classification of ( $\left.\pi, m\right)$-complexes, J. Pure Appl. Algebra 7 (1976), 249-282.

[Sc] J. Schafer, Finite complexes and integral representations. II, Canad. Math. Bull. 27 (1983), 1-7.

[M] W. Metzler, Uber den Homotopietyp zweidimensionaler $C W$-complexe..., J. Reine Angew. Math. 285 (1976), 7-23.

[S] A. Sieradski, A semigroup of simple homotopy type, Math. Z. 153 (1977), 135-148.

[DS] M. Dyer and A. Sieradski, Trees of homotopy types of two-dimensional CW-complexes, Comment Math. Helv. 48 (1973), 31-44.

Department of Mathematics, University of Oregon, Eugene, Oregon 97403 